\title{
Enteroparasitoses em uma comunidade fechada. I - Estudo do solo como elo de transmissão em um orfanato de Manaus, Amazonas, Brasil (")
}

\author{
Maria de Fátima da Silva Pinheiro ("*) \\ Mauro Célio de Almeida Marzochi (***) \\ Rodolfo Giugliano (**) \\ Loreny Gimenes Giugliano ("*)
}

\section{Resumo}

Por meio de métodos parasitológicos, baseados na centrifugo- flutuação em sulfato de zinco, os AA estudaram a prevalência das enteroparasitoses em 49 crianças de um orfanato da cidade de Manaus e, paralelamente, a contaminação fecal de seu solo nas principais áreas de recreação. Os resultados mostraram que $100 \%$ das crianças examinadas apresentaram uma ou mais espécies de parasitas e que $90 \%$ das amostras do solo estavam contaminadas tanto por cistos de protozoários como por ovos de helmintos parasitas.

\section{INTRODUÇÃO}

Em muitas regiões, as parasitoses intestinais representam problemas médico-sanitários de grande importância pela freqüência com que ocorrem e, especialmente, pela possibilidade de determinarem acometimentos orgânicos capazes, às vezes, de incapacitarem os indivíduos atingidos (Amato Neto et al,, 1968). Tal situação é bastante conhecida em nosso País.

Em nosso meio, Costa (1949) verificou nas cidades de Cametá, Abaetetuba e Monte Alegre, no Estado do Pará, e de Itacoatiara, no Estado do Amazonas, que era de $37,6 \%$ e $25,4 \%$, respectivamente, a prevalência da ancilostomose; Causey et al. (1947), em Belém, encontraram $42,3 \%$ de ancilostomóticos em individuos com menos de 15 anos e $46,5 \%$ em indivíduos com idade superior; Oliveira (1959), em Manaus, Amazonas, verificou ser de 14,6\% a prevalência para Entamoeba histolytica e de Giardia lamblia na população.

Em trabalho anterior, um dos autores, Pinheiro et al. (1976), em dois bairros de Ma- naus, obteve $74 \%$ de Trichocephalus trichiurus, $58 \%$ de Ascaris lumbricoides, $53 \%$ de Ancylostomidae, $9 \%$ de Strongyloides stercoralis, $17 \%$ de Giardia lamblia e 3\% de Entamoeba histolytica, em exames coproparasitológicos.

E sabido que a epidemiologia das parasitoses nas populações humanas depende de fatores bionômicos e ecológicos que afetam a sobrevivência e propagaçẵo dos agentes patogênicos. Concomitantemente, isso é grandemente influenciado pelas condições econômicas e sociais da população, pelo padrão sanitário e de higiene da comunidade e pelas condições de trabalho e comportamento de seus membros. Contudo, esse quadro global de morbidade e propagação das parasitoses possui uma epidemiologia especial em populações confinadas e em instituiçōes nas quais a disseminação e persistência dessas doenças são pouco influenciadas pelos fatores externos e, apenas levemente, afetadas por alterações climáticas e sazonais (Yoeli et al., 1972).

O conhecimento de peculiaridades regionais e locais envolvidas na disseminação dos enteroparasitas é importante para adoção mais efetiva das medidas de educação sanitária, prevenção, terapêutica, acompanhamento e vigilância das condições de saúde de determinada comunidade.

O objetivo dessa linha de pesquisa é tentar conhecer a história natural das enteroparasitoses em uma população estável, de regiäo tropical, através do estudo de suas prevalências assim como dos principais fatores envolvidos na disseminação das mesmas.

\footnotetext{
(:) - Pesquisa financiada pelo CNPq. Projeto $n 9$ 2017/201, do INPA.

(*) - Instituto Nacional de Pesquisas da Amazônia, Manaus.

(••) - Universidade Estadual de Londrina, Paraná.
} 


\section{MATERIAL E MÉTODOS}

Foi escolhido um orfanato do perímetro urbano da cidade de Manaus, abrigando crianças e adolescentes de ambos os sexos, dirigido por uma instituição filantrópica.

As idades dos internos variam de 2 a 17 anos e a proporção entre os sexos é semeIhante. Os internos raramente deixam os limites da instituição. As condiçōes do orfanato são insatisfatórias: elevado número de internos dormindo no mesmo aposento; banheiros e instalaçōes sanitárias em pequerio número, nāo permitindo obedecer normas satisfatórias de higiene; áreas de recreação cobertas per vegetação, permitindo que as crianças evacuem nas proximidades

No periodo de setembro a novembro de 1976 foram examinadas amostras de fezes de 49 internos, de ambos os sexos e todos os grupos etários.

Para coleta de material foram distribuídos recipientes plásticos de boca larga, com capacidade de $100 \mathrm{~cm}^{3}$, devidamente identificados, recolhidos e examinados em grupo de cinco.

Os exames foram realizados na Divisão de Ciências Médicas (Departamento de Doenças Gastrointestinais) do Instituto Nacional de Pesquisas da Amazônia (INPA). Foi utilizado apenas o método da centrifugo-flutuação em sulfato de zinco de Faust et al. (1939) que segundo Lima et al. (1969), é tido como um dos mais eficientes para a recuperação de cistos de protozoários e ovos de helmintos das fezes.

Conhecidos os resultados, as crianças foram submetidas a exame clínico e medicadas devidamente por um médico especializado.

Em seguida, foram colhidas 20 amostras de terra pela raspagem da superfície do soto numa profundidade de aproximadamente $10 \mathrm{~mm}$ e, com auxílio de espátulas metálicas, acondicionadas em tubos de ensaio de $18 \times 180 \mathrm{~mm}$. Os pontos de coleta foram equidistantes, em locais sombreados, nas princicipais áreas de recreação do orfanato.

Para o mdespreendimento dos ovos e cistos dos detritos do solo, adicionou-se a cada amostra uma solução de $\mathrm{NaOH}$ a $0,10 \mathrm{~N}$, agi- tando-se fortemente em um agitador elétrico tipo "Mixer" e deixando-se em repouso durante 15 minutos. Em seguida, o sobrenadante era desprezado e o sedimento obtido ressuspendido em solução de sulfato de zinco com densidade de 1,180, agitado e transferido para um tubo de $10 \mathrm{~cm}^{3}$, centrifugado e examinado ao microscópio, conforme o preconizado por Faust et al. (1939).

\section{RESUltados E Discussão}

Observamos que dentre os 49 individuos examinados $49(100,0 \%)$ tiveram exame positivo para, pelo menos, um parasita. Quanto ao sexo, tivemos $25(51,0 \%)$ individuos do sexo masculino e $24(49,0 \%)$ do sexo feminino.

A frequeência de helmintos e protozoários encontrados em relação ao sexo e grupos etários é mostrada na Tabela $\mathrm{I}$.

Entre os helmintos, o parasita mais freqüente foi o Trichocephalus trichiurus em $92,0 \%$ dos indivíduos do sexo masculino e $87,5 \%$ do sexo feminino; seguindo-se o Ascaris lumbricoides em $68,0 \%$ do sexo masculino e $79,2 \%$ do sexo feminino; o Ancylostomidae, $80.0 \%$ de masculinos e $62,5 \%$ de femininos: o Strongyloides stercoralis, $4.1 \%$ de masculinos e $20,8 \%$ de femininos; o Enterobius vermiculares, $16.7 \%$ de feminino; e, dentre os protozoários, o de maior prevalência foi a $E n$ tamoeba coli em $44,0 \%$ dos individuos do sexo masculino e $33,3 \%$ do sexo feminino, seguindo-se a Giardia lamblia em $48,0 \%$ de masculinos e $20,8 \%$ do sexo feminino; a lodamoeba butschlii, $8.0 \%$ de masculinos e $8.3 \%$ de femininos; e a Entamoeba histolytica, $8.0 \%$ de masculinos e $4,2 \%$ de femininos.

A Tabela II mostra a freqüência de cistos e ovos de parasitas intestinais encontrados no solo, evidenciando que o $T$. trichiurus também predominou entre os helmintos em $45.0 \%$ das 20 amostras analisadas, seguindo-se do A. lumbricoides em 40,0\%, o Ancylostomidae, $30,0 \%$; o S. stercoralis, $25.0 \%$; e, dentre os protozoárıos, destacou-se a Giardia lamblia, $20,0 \%$; a Entamoeba coli, $15,0 \%$ e a Entamoe. ba histolytica $10,0 \%$. 
TABELA I - Prevalência específica de enteroparasitas, segundo idade e sexo, em um orfanato de Manaus, Amazonas, Brasil - 1976

\begin{tabular}{|c|c|c|c|c|c|c|c|c|c|c|c|}
\hline $\begin{array}{c}\text { Grupo etário } \\
\text { (Anos) }\end{array}$ & \multicolumn{2}{|c|}{$2-5$} & \multicolumn{2}{|c|}{$6-9$} & \multicolumn{2}{|c|}{$10-13$} & \multicolumn{2}{|c|}{$14-17$} & \multicolumn{2}{|c|}{ T O T A L } & $\begin{array}{l}\text { TOTAL } \\
\text { GERAL }\end{array}$ \\
\hline $\begin{array}{l}\text { Sexo } \\
\text { N.० }\end{array}$ & $\stackrel{\delta}{N=2}$ & $\stackrel{q}{N}=0$ & $\stackrel{\delta}{N=5}$ & $\stackrel{q}{N=7}$ & $\stackrel{\delta}{\delta}=10$ & $\stackrel{9}{N=14}$ & $\stackrel{\delta}{N=8}$ & $\stackrel{\stackrel{9}{1}}{\mathbf{N}=3}$ & $\mathbf{N} \stackrel{\hat{\sigma}}{=} 25$ & $\stackrel{\stackrel{9}{7}}{N=24}$ & $N=49$ \\
\hline Parasitos & POS. & POS. & POS. & POS. & POS. & POS. & POS. & POS. & POS. (\%) & POS. (\%) & POS. $(\%)$ \\
\hline T. trichiurus & 2 & - & 5 & 5 & 10 & 13 & 6 & 3 & $23(92,0)$ & $21(87,5)$ & $44(89,8)$ \\
\hline A. lumbricoides & 1 & $\rightarrow$ & 4 & 6 & 6 & 11 & 6 & 2 & $17(68,0)$ & $19(79,2)$ & $36(73,5)$ \\
\hline Ancylostomidae & 一 & $\rightarrow$ & 3 & 3 & 9 & 10 & 8 & 2 & $20(80,0)$ & $15(62,5)$ & $35(71,4)$ \\
\hline S. stercoralis & - & $\rightarrow$ & $\rightarrow$ & $\rightarrow$ & 1 & 4 & $\rightarrow$ & 1 & $1(4,1)$ & $5(20,8)$ & $6(12,2)$ \\
\hline E. vermiculares & $\rightarrow$ & $\rightarrow$ & $\rightarrow$ & - & - & 3 & $\rightarrow$ & $\rightarrow$ & - $\quad-$ & $4(16,7)$ & $4 \quad(8,2)$ \\
\hline E. coli & 1 & $\rightarrow$ & 2 & 4 & 5 & 3 & 3 & 1 & $11(44,0)$ & $8(33,3)$ & $19(38,8)$ \\
\hline G. lamblia & 2 & $一$ & 3 & 3 & 4 & $\rightarrow$ & 3 & 2 & $12(48,0)$ & $5(20,8)$ & $17(34.7)$ \\
\hline I. butschlii & $\rightarrow$ & $\rightarrow$ & - & 一 & 1 & 1 & 1 & 1 & $2 \quad(8,0)$ & $2 \quad(8,3)$ & $4 \quad(8,2)$ \\
\hline E. histolytica & $\rightarrow$ & - & $一$ & $\rightarrow$ & 1 & $\rightarrow$ & 1 & 1 & $2 \quad(8,0)$ & $1 \quad(4,2)$ & $3 \quad(6,1)$ \\
\hline
\end{tabular}

TABELA II - Freqüência de ovos e cistos de ente. roparasitas em 20 amostras do solo de um orfana. to de Manaus, Amazonas, Brasil - 1976

\begin{tabular}{lcc}
\hline P A R A S T T S & POS. & $(\%)$ \\
\hline T. trichiurus & 9 & $(45,0)$ \\
A. lumbricoides & 8 & $(40,0)$ \\
Ancylostomidae & 6 & $(30,0)$ \\
S. stercoralis & 5 & $(25,0)$ \\
G. lamblia & 4 & $(20,0)$ \\
E. coli & 3 & $(15,0)$ \\
E. histolytica & 2 & $(10,0)$ \\
\hline TO T A L & 18 & $(90,0)$ \\
\hline
\end{tabular}

É digno de nota que as presencas de parasitas não menos importantes como o Strongyloides stercoralis, Enterobius vermicularis, Taenia sp, etc., estăo subestimadas em virtude da não utilização de metodologia mais específica, tanto em relação a infestação dos internos, como a contaminação do solo.

Apesar da relativa baixa especificidade do método empregado, em relação a ovos e larvas de helmintos, o indice de parasitismo observădo entre os internos $(100,0 \%)$ foi muito elevado quando comparado com os resultados obtidos em levantamentos anteriores realiza- dos em bairros de baixa condição sócio-econômica e sanitária de Manaus, por Pinheiro et al. (1976).

Este fato sugere que fatores ligados ao próprio orfanato (ambiente fechado, facilidade de contato inter-humano e tendência a promiscuidade) devem estar interferindo na prevalência desses enteroparasitas, principalmente os transmitidos pelo solo (Moretti et al., 1974). Isso é reforçado pelo alto indice de contaminação do solo nas áreas de recreação, além do paralelismo existente entre a ordem de fre. qüência dos parasitos encontrados nos exames coprológicos e no solo.

A relativa alta prevalência de Giardia lamblia $(37,7 \%)$ e Entamoeba histolytica $(8.2 \%)$, assim como de protozoários comensais como a Entamoeba coli $(38,8 \%)$ e a lodamoeba butschlii $(8,2 \%)$ sugere que esteja ocorrendo, de alguma forma, contaminação fecal da água ou de alimentos consumidos crus (Moretti et al., 1974: Marzochi, 1975), o que será motivo de estudos futuros.

O presente estudo vem reforçar, de certa forma, a importância do solo na disseminação de alguns enteroparasitas uma vez que, como refere Vinha (1965), o solo se comporta como um hospedeiro intermediário. Recebe fezes 
ou águas contaminadas por parasitas, em estádios não infestantes, oferecendo-lhes condições de desenvolvimento, protegendo-os, em estádios infestantes, durante certo período para, posteriormente, transmití-los ao homem.

\section{AgradeCLMENTOS}

A Srta. Ana Maria de Souza Lira que nos auxiliou nas coletas de amostras de solo e preparação das soluções químicas empregadas.

\section{SUMMARY}

During the months of September through November a parasitological investigation was made from fecal samples of 49 children and 20 soil samples of an orphanage in Manaus, Amazonas, Brazil. The specimens were concentrated by Faust's method, and the authors prepared the following index of intestinal parasites in these individuals. These results were obtained.

Helminths: Trichocephalus trichiurus $89,0 \%$, Ascaris lumbricoides $73,5 \%$; Ancylostomidae $71,4 \%$; Strongyloides stercoralis $12,2 \%$ and Enterobius vermiculares $8,2 \%$.

The highest incidence of protozoan parasites was of Entamoeba coli $38,8 \%$, Giardia lamblia $34,7 \%$, Iodamoeba butschlii $8,2 \%$ and Entamoeba histolytica $6,1 \%, 90 \%$ of the soil samples were contaminated.

\section{BIBLIOGRAFIA CITADA}

Amato Neto, R.; Campos, R.; \& Ferreira, C.S.

1968 - Diagnóstico das parasitoses intestinais pelo exame das fezes. 3 ed. São Paulo, Artes Médicas Ltda. $128 \mathrm{p}$. il.

CAUSEY, O.R.

1947 - Incidência de parasitos intestinais do homem na cidade de Belém, Pará e vizinhanças. Revista do Serviço Especial de Saúde Pública, 1(12) : 221-233.
Costa, O.R.

1949 - Contribuição ao conhecimento de incidências dos helmintos e protozoários intestinais na Amazônia. Tese, 123 pp. Trop. Rev. Veterinária, Belém.

Faust, E.C.; SAwITZ, W.; TовIE, J.; ОDом, V.; PERES, C. \& LiNCICOME, D.R.

1939 - Comparative efficiency of various technics for the diagnosis of protozoa and helminths in fezes. J. Parasit, 25 : 241-262.

Lima, A.O.; SOARes, J.B.; Greco, J.B.; Galizzi, J. \& CANÇADO, J.R.

1969 - Métodos de laboratório aplicados à clínica. 4 ed., Rio de Janeiro, Koogan S.A. S.A. 653 p. il.

MARZOCHI, M.C. DE A.

1975 - Estudo epidemiológico da poluição por enteroparasitas em áreas de horticultura da cidade de Ribeirão Preto, SP, Brasil (Tese - Universidade Estadual de Londrina).

Moretti, I.G.; Chieffi, P.P.; Nakagawa, E.; Gomes, A. DE C. \& FOIZER, A.C.

1974 - Contribuição ao estudo da história natural de enteroparasitoses em uma comunidade fechada. I - Prevalência de enteroparasitas em uma comunidade fechada. Rev. Soc. Bras. Med. Trop., 8(1): 41-44.

Oliverra, W.R.

1959 - Contribuição ao estudo coprológico na cidade de Manaus. Brasil Médico, 73(7/28):123-125.

Pinheiro, M.F.S.; VAsconcelos, J.C. \& Wendell, D.E.

1976 - Contribuição ao estudo de parasitos in. testinais em dois bairros de Manaus. Amazonas. Acta Amazonica, 6(1):67-73.

VINHA, C

1965 - Fundamentos e importância das campanhas contra os geo-helmintos no Brasil. Rev. Bras. Malar., 17:379-406.

Yoeli, M.; MOST, H.; HAMmond, J. \& SCHernesson

1972 - Parasitic Infections in a closed com munity - Results of 10-year survey in Willowbrook Stat School. Trans. R. Soc. Trop. Med. Hyg. 66(5):764-766. 